\title{
Transition between wurtzite and zinc-blende GaN: An effect of deposition condition of molecular-beam epitaxy
}

\author{
B. M. Shi, M. H. Xie, ${ }^{\text {a) }}$ and H. S. Wu \\ Department of Physics, The University of Hong Kong, Pokfulam Road, Hong Kong, China \\ N. Wang \\ Department of Physics, Hong Kong University of Science and Technology, Clear Water Bay, Kowloon, \\ Hong Kong, China \\ S. Y. Tong \\ Department of Physics and Materials Science, City University of Hong Kong, Kowloon Tang, Hong Kong, \\ China
}

(Received 6 July 2006; accepted 20 August 2006; published online 13 October 2006)

\begin{abstract}
GaN exists in both wurtzite and zinc-blende phases and the growths of the two on its (0001) or (111) surfaces are achieved by choosing proper deposition conditions of molecular-beam epitaxy (MBE). At low substrate temperatures but high gallium fluxes, metastable zinc-blende $\mathrm{GaN}$ films are obtained, whereas at high temperatures and/or using high nitrogen fluxes, equilibrium wurtzite phase GaN epilayers resulted. This dependence of crystal structure on substrate temperature and source flux is not affected by deposition rate. Rather, the initial stage nucleation kinetics plays a primary role in determining the crystallographic structures of epitaxial GaN by MBE. (C) 2006 American Institute of Physics. [DOI: 10.1063/1.2360916]
\end{abstract}

It is known that $\mathrm{GaN}$ exists in both wurtzite $(w)$ and zinc-blende $(\mathrm{zb})$ structural phases, where the former represents the equilibrium and the latter is metastable at normal conditions. ${ }^{1}$ The formation energies of the two polymorphs are very close ${ }^{2}$ therefore it is often that the two structures coexist in an epifilm grown by metal organic vapor phase epitaxy (MOVPE) or molecular-beam epitaxy (MBE)., ${ }^{3,4}$ Zinc-blende $\mathrm{GaN}$ has drawn increasing attention recently due to its superior electronic and optical properties over $w$-GaN. ${ }^{5}$ For example, the mobility of electrons and holes in a zb-GaN film is intrinsically higher than that in a $w$-GaN layer due to lower phonon scattering in cubic crystals. ${ }^{6}$ The easier cleavage of zb-GaN also makes it attractive for device applications such as lasers. In addition, there is a type-II band alignment at $\mathrm{zb}-\mathrm{GaN} / w-\mathrm{GaN}$ interface, which may find applications for improved device performances. ${ }^{7,8}$

Conventionally, one uses cubic symmetrical substrates such as $\mathrm{GaAs}(100)$ and $\mathrm{Si}(100)$ to stabilize and grow $\mathrm{zb}-\mathrm{GaN} .{ }^{4} \mathrm{On}$ the other hand, it was also found that $\mathrm{zb}-\mathrm{GaN}$ may grow even on substrates with hexagonal symmetry such as sapphire(0001), ${ }^{9-11} \mathrm{SiC}(0001),{ }^{12}$ and $\mathrm{GaAs}(111){ }^{13}$ In some cases, zb-GaN films were induced by doping, ${ }^{11,12}$ while in others they were caused by choosing proper deposition conditions. ${ }^{9,10,13}$ If $\mathrm{zb}-\mathrm{GaN}$ grows on cubic substrates to reduce interfacial energy, it is mysterious why (111)-oriented zb-GaN films can grow on surfaces of hexagonal symmetry. After all, zinc-blende $\mathrm{GaN}$ is not the equilibrium phase and the growth of it would cost extra energy. It would be of great fundamental interests as well as of practical relevances to understand such a growth phenomenon.

In this work, we first establish the MBE conditions that favor zb-GaN and $w$-GaN films, respectively. Then we show that the initial stage nucleation kinetics is of primary importance in determining the crystallographic structures of $\mathrm{GaN}$

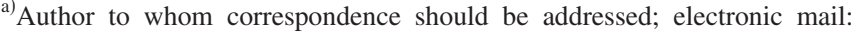
mhxie@hkusua.hku.hk
}

epilayers. The possible reasons for preferential nucleation of $\mathrm{zb}-\mathrm{GaN}$ at low temperature and excess Ga flux are also discussed.

The experiments were carried out in a multichamber ultrahigh vacuum system, where the MBE reactor was connected with some surface probing tools via vacuum interlocks. ${ }^{14}$ In MBE, conventional effusion cells for gallium $(\mathrm{Ga})$ and a radio-frequency plasma unit for nitrogen $(\mathrm{N})$ were installed. It also contained the facility of reflection highenergy electron diffraction (RHEED) operating at $10 \mathrm{keV}$. Nominally flat $6 \mathrm{H}-\mathrm{SiC}(0001)$ was used as the substrate, upon which a thick ( $\geqslant 3000 \AA$ ) $\mathrm{GaN}$ buffer layer was grown at $\geqslant 620{ }^{\circ} \mathrm{C}$ using an excess Ga flux. The buffer layer surface was smooth as reflected by streaky RHEED patterns and confirmed by scanning tunneling microscopy (STM). It was of wurtzite phase with Ga polarity according to surface reconstruction observations. ${ }^{15}$ Having completed the buffer layer deposition, the substrate temperature and/or source fluxes were adjusted before resuming $\mathrm{GaN}$ deposition at the new conditions of MBE. For some, submonolayers of GaN were deposited followed by thermal quenching of the surfaces for subsequent STM examinations, while in others thick layers of $\mathrm{GaN}$ were grown for ex situ transmission electron microscopy (TEM) experiments. For STM, the tunneling current was $0.1 \mathrm{nA}$ and the sample bias was $-2.5 \mathrm{~V}$, while for conventional and high resolution (HR) TEM experiments, a Philips CM200FEG TEM microscope was used operating at $200 \mathrm{keV}$. Cross-sectional TEM specimens were prepared by the standard method of mechanical polishing and argon ion milling.

Figure 1 summarizes the temperature and flux dependences of the crystal structures of GaN. The general feature emerged from our experiments is that when the temperature is low and the flux ratio $R$ between $\mathrm{Ga}$ and $\mathrm{N}$ sources is high $(R>1)$, zb-GaN films will be obtained. At other conditions, films with the hep stacking will result. Here the fluxes of nitrogen are referred to as the effective values, which were 


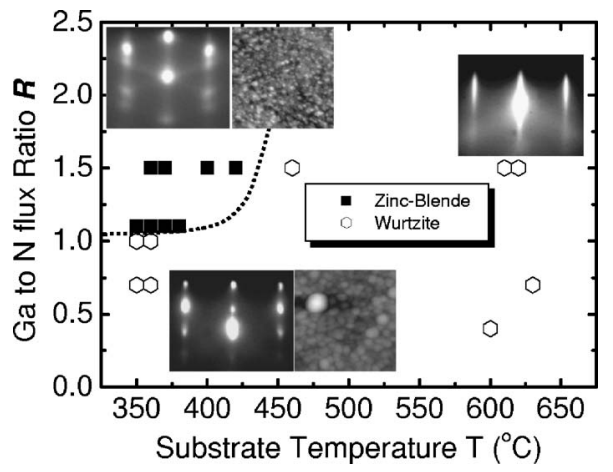

FIG. 1. "Phase diagram" summarizing the crystal structures of GaN grown by MBE under different temperature and flux conditions. The insets show the corresponding RHEED patterns and STM micrographs (size: 200 $\times 200 \mathrm{~nm}^{2}$ ) of the surfaces obtained at different conditions. The dotted line is drawn to indicate the phase boundary between $\mathrm{zb}-\mathrm{GaN}$ and $w-\mathrm{GaN}$.

calibrated by film growth rate in the $\mathrm{N}$-limited regimes by RHEED. ${ }^{14}$ The insets in Fig. 1 show representative RHEED patterns and STM micrographs of GaN grown under different conditions. Generally, low deposition temperatures or high $\mathrm{N}$ fluxes lead to rough surfaces and the corresponding RHEED patterns are spotty. From the symmetry of the transmission RHEED spots, one may infer about the crystal structure of the film as shown in Ref. 9. On the other hand, for growth at high temperatures and using excess Ga fluxes, the film surfaces are usually smooth and the RHEED patterns are streaky. For such films, TEM has been used to determine their crystal structures.

Figure 2 shows conventional and HRTEM micrographs of a sample grown consecutively at high $\left(620^{\circ} \mathrm{C}\right)$ and low $\left(420{ }^{\circ} \mathrm{C}\right)$ temperatures. It reveals the structural change from wurtzite to zinc-blende upon temperature reduction, demonstrating that $\mathrm{zb}-\mathrm{GaN}$ grows even on $\mathrm{GaN}(0001)$ given the proper conditions of MBE. It also reveals that except at the interface, which is highly faulted, the top $\mathrm{zb}-\mathrm{GaN}$ and bottom $w$-GaN layers are of reasonably high phase purity. However, we also wish to point out that the density of extended defects, such as dislocations, stacking faults, and twin boundaries, is usually higher in zb-GaN layers than in wurtzite films. This is probably caused by the unfavorably low growth temperature necessary for $\mathrm{zb}-\mathrm{GaN}$ formation.

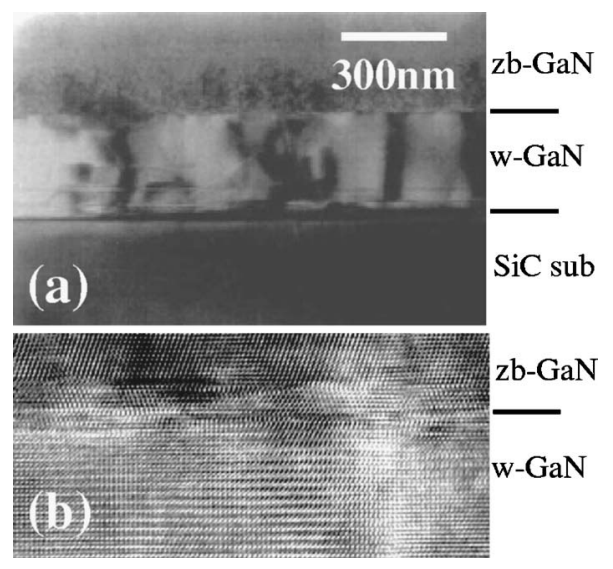

FIG. 2. (a) Conventional and (b) HRTEM micrograph of a sample grown at two different temperatures. The bottom layer was grown at $620^{\circ} \mathrm{C}$ and the top layer was at $420^{\circ} \mathrm{C}$. The flux ratio between $\mathrm{Ga}$ and $\mathrm{N}$ was 1.5 throughout.
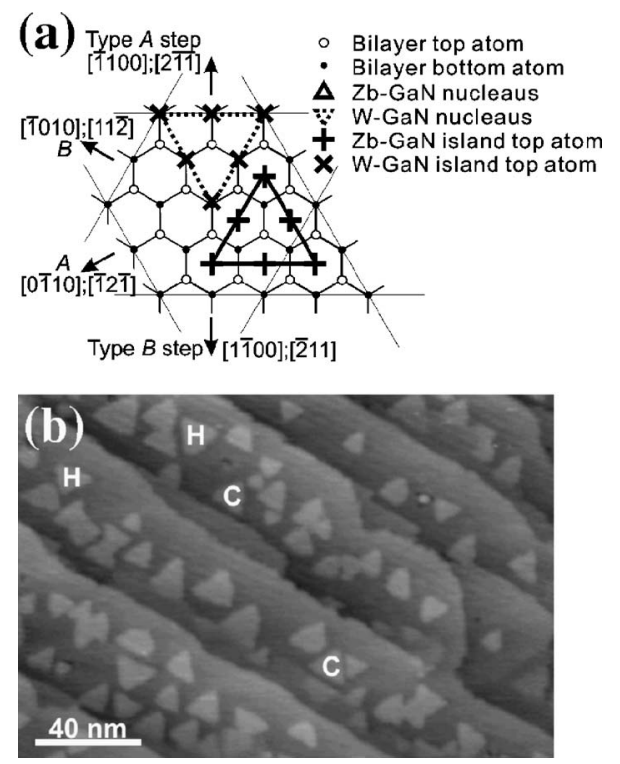

FIG. 3. (a) Schematic diagram showing the surface of $w$-GaN $(0001)$ or $\mathrm{zb}-\mathrm{GaN}(111)$. Triangular $\mathrm{zb}-\mathrm{GaN}$ and $w$-GaN nuclei are drawn to show their opposite orientations grown on the same surface. (b) STM image of a surface following submonolayer $\mathrm{GaN}$ deposition at $340{ }^{\circ} \mathrm{C}$. Some of the islands representing $\mathrm{zb}-\mathrm{GaN}$ and $w$-GaN nuclei are marked by $\mathrm{C}$ and $\mathrm{H}$, respectively.

It is noted that similar conditions of excess $\mathrm{Ga}$ and/or low temperature have been identified also by others to favor zb-GaN growth. ${ }^{9,10,13,16}$ Hence it appears that this phenomenon of $\mathrm{GaN}$ growth is robust, reflecting some underlying physical mechanisms rather than growth errors or fluctuations. ${ }^{17}$ To investigate the mechanism, we firstly examined the effect of deposition rate where the growth of $\mathrm{GaN}$ was conducted at different growth rates ranging from 0.05 to $0.42 \AA / \mathrm{s}$. No apparent deviation from the results of Fig. 1 was observed, though higher growth rates generally led to higher densities of extended defects in films. A known mechanism of metastable phase crystal growth is the Ostwald step rule, where a stable phase crystal grows via some intermediate metastable phases at high supersaturation of the system. ${ }^{18}$ This mechanism was shown to originate from the higher nucleation rate of the metastable phase than that of the equilibrium structure. ${ }^{18}$ Varying the temperature and varying the source fluxes both lead to changes of supersaturation; so if the temperature change has induced a phase transition of $\mathrm{GaN}$, a similar effect may be expected by changing the flux or growth rate. ${ }^{19}$ This is, however, not observed in this study for the range of growth rates experimented. ${ }^{20}$

In an earlier study by TEM of low-temperature buffer layers grown by MOVPE, faceting of $\mathrm{zb}-\mathrm{GaN}$ islands was noted. ${ }^{16}$ Based on this, it was suggested that $\mathrm{zb}-\mathrm{GaN}$ formation under excess $\mathrm{Ga}$ was not caused by preferential nucleation of $\mathrm{zb}-\mathrm{GaN}$, but that the condition promoted rapid growth of zb-GaN nuclei coexisted with $w$-GaN nuclei. ${ }^{17,21}$ To investigate the role of initial stage nucleation on $\mathrm{zb}-\mathrm{GaN}$ growth, we examined by STM the nucleation island shape and orientations. Figure 3 shows an example of the surface prepared at $340{ }^{\circ} \mathrm{C}$, revealing triangular islands residing on terraces separated by double bilayer steps. As detailed in Ref. 22 , the doubly bunched step structure is characteristic of a wurtzite crystal surface. Together with the triangular islands, it reflects the anisotropic growth rates of surface steps. As 
TABLE I. Number of zb-GaN and $w$-GaN nuclei on $w$-GaN(0001) surface following submonolayer deposition at different temperatures.

\begin{tabular}{cccc}
\hline \hline $\begin{array}{c}\text { Temperature } \\
\left({ }^{\circ} \mathrm{C}\right)\end{array}$ & $\begin{array}{c}\text { zb-GaN nuclei } \\
\left(N_{\mathrm{zb}}\right)\end{array}$ & $\begin{array}{c}w \text {-GaN nuclei } \\
\left(N_{w}\right)\end{array}$ & $\begin{array}{c}\text { Ratio of } \\
N_{w} / N_{\mathrm{zb}}\end{array}$ \\
\hline 340 & 649 & 464 & 0.71 \\
360 & 421 & 312 & 0.74 \\
400 & 1042 & 773 & 0.74 \\
460 & 18 & 98 & 5.44 \\
\hline \hline
\end{tabular}

depicted in Fig. 3(a), the type- $A$ step has two dangling bonds per edge atom whereas the type- $B$ step has only one bond per atom. ${ }^{22}$ The two steps might thus have different capabilities in capturing adatoms and so grow at different rates. ${ }^{22,23} \mathrm{On}$ both $w$-GaN(0001) and zb-GaN(111), type- $A$ and $-B$ steps alternate upon turning $60^{\circ}$, so the anisotropic growth rates of the two steps naturally lead to triangular islands. ${ }^{22}$ On the other hand, steps change character from $A$ to $B$ or from $B$ to $A$ upon descending single or odd number of atomic steps for a wurtzite film, so the anisotropic step growth rates between $A$ and $B$ result in double step bunching. For growth of a zinc-blende layer, however, since steps are of the same type along a given crystallographic direction, no double step bunching is expected. ${ }^{22}$ Based on the above, we may identify the islands in Fig. 3(b) to be nuclei of zb-GaN or $w$-GaN by noting their orientations. As illustrated in Fig. 3(a), if atoms in an island occupy the $T 4$ sites, representing the hop stacking with respect to the buffer layer lattices, the island becomes a nucleus of wurtzite GaN. One of the triangle vortices points to the ascending double bilayer step of the terrace. Oppositely, if atoms occupy the $H 3$ sites, it represents a nucleus of $\mathrm{zb}-\mathrm{GaN}$ and its orientation will be that pointing to the descending step. After distinguishing between $\mathrm{zb}-\mathrm{GaN}$ and $w$-GaN islands, we count their numbers from numerous STM images similar to Fig. 3(b). Table I summarizes the results for deposition on $w$-GaN(0001) surfaces. For those nucleated on $\mathrm{zb}-\mathrm{GaN}(111)$, as the surfaces of grown $\mathrm{zb}-\mathrm{GaN}$ are rough, such data are not yet available. Nevertheless, the results of Table I already reveal a clear transition from $w$-GaN dominated to $\mathrm{zb}-\mathrm{GaN}$ dominated nucleation as the temperature is lowered. So, it is very likely that the growths of $\mathrm{zb}-\mathrm{GaN}$ at low temperature and excess Ga but $w$-GaN at other conditions are directly related to preferential nucleation of islands of the respective type at the early stages of deposition.

Another feature seen from Table I is that there is a huge asymmetry about the island ratio of $w-\mathrm{GaN}$ to $\mathrm{zb}-\mathrm{GaN}$ at low versus high temperatures (e.g., at temperatures below $420{ }^{\circ} \mathrm{C}$, the ratio is about 0.7 , whereas at $460{ }^{\circ} \mathrm{C}$, the ratio is $\sim 5.4$ ). If such ratios propagate for growth of consecutive layers, one expects that the transition from $w-\mathrm{GaN}$ to $\mathrm{zb}-$ $\mathrm{GaN}$ is not as sharp as that from zb-GaN to $w$-GaN upon changing the MBE conditions. This is consistent with the experiments of Yang et $_{\text {al. }}{ }^{13}$

Finally, it remains a question why low temperatures and high $\mathrm{Ga}$ fluxes favor $\mathrm{zb}-\mathrm{GaN}$ nucleation. Considering the sensitive dependence on deposition conditions of MBE, we believe that it is controlled by atomic surface kinetics involving surface excess Ga. Apart from the Ostwald step rule mentioned earlier, one possibility is the preferential adsorption of atoms on $\mathrm{H3}$ sites of the surface. In a previous study of adsorption on $\mathrm{Si}(100)$, it was shown that surface sites with local minima could be more favorable for absorption than the equilibrium sites with global energy minimum. ${ }^{24}$ If such atoms are trapped in such metastable sites by, e.g., low substrate temperature, they will act as the nuclei for subsequent material growth and so the metastable phase of a crystal will result. Unfortunately, such studies of atom adsorption on $\mathrm{GaN}$ are not yet available.

To summarize, both $\mathrm{zb}-\mathrm{GaN}$ and $w-\mathrm{GaN}$ films are achieved on $w$-GaN(0001) or zb-GaN(111) surfaces by tuning the conditions of MBE. The necessary conditions for metastable $\mathrm{zb}-\mathrm{GaN}$ growth are low temperature and excess Ga flux. We further show that preferential nucleations of $\mathrm{zb}-$ $\mathrm{GaN}$ or $w$-GaN islands are of primary importance in determining the crystal structures of the grown film.

This work is supported by the Research Grant Council of Hong Kong Special Administrative Region, China, under Grant No. HKU7048/04P.

${ }^{1}$ S. Strite and H. Morkoc, J. Vac. Sci. Technol. B 10, 1237 (1992).

${ }^{2}$ A. F. Wright, J. Appl. Phys. 82, 5259 (1997).

${ }^{3}$ M. Mizuta, S. Fujida, Y. Matsumoto, and T. Kawamura, Jpn. J. Appl. Phys., Part 2 25, L945 (1986).

${ }^{4}$ K. H. Ploog, O. Brandt, H. Yang, B. Yang, and A. Trampert, J. Vac. Sci. Technol. B 16, 2229 (1998).

${ }^{5}$ H. Okumura, K. Ohta, G. Feuillet, K. Balakrishnan, S. Chichibu, H. Hamaguchi, P. Hacke, and S. Yoshida, J. Cryst. Growth 178, 113 (1997).

${ }^{6}$ P. Das and D. K. Ferry, Solid-State Electron. 19, 851 (1976).

${ }^{7}$ Z. Z. Bandic, T. C. MacGill, and Z. Ikonic, Phys. Rev. B 56, 3564 (1997).

${ }^{8}$ X. H. Lu, P. Y. Yu, M. H. Xie, L. X. Zheng, S. J. Xu, and S. Y. Tong, Appl. Phys. Lett. 82, 1033 (2003).

${ }^{9}$ H. Okumura, K. Balakrishnan, H. Hamaguchi, T. Koizumi, S. Chichibu, H. Nakanishi, T. Nagatomo, and S. Yoshida, J. Cryst. Growth 189/190, 364 (1998).

${ }^{10}$ T. Kurobe, Y. Sekiguchi, J. Suda, M. Yoshimoto, and H. Matsunami, Appl. Phys. Lett. 73, 2305 (1998)

${ }^{11}$ Y. Zhao, C. W. Tu, I.-T. Bae, and T.-Y. Seong, Appl. Phys. Lett. 74, 3182 (1999).

${ }^{12}$ Y. Cui, V. K. Lazorov, M. M. Goetz, H. Liu, D. P. Robertson, M. Gajdardziska-Josifovska, and L. Li, Appl. Phys. Lett. 82, 4666 (2003).

${ }^{13}$ J. W. Yang, J. N. Kuznia, Q. C. Chen, M. Asif Khan, T. George, M. De Graef, and S. Mahajan, Appl. Phys. Lett. 67, 3759 (1995).

${ }^{14}$ S. M. Seutter, M. H. Xie, W. K. Zhu, L. X. Zheng, Huasheng Wu, and S. Y. Tong, Surf. Sci. 445, L71 (2000).

${ }^{15}$ A. R. Smith, R. M. Feenstra, D. W. Greve, M.-S. Shin, M. Skowronski, J. Neugebauer, and J. E. Northrup, Appl. Phys. Lett. 72, 2114 (1998).

${ }^{16}$ X. H. Wu, D. Kapolnek, E. J. Tarsa, B. Heying, S. Keller, B. P. Keller, U. K. Mishra, S. P. DenBaars, and J. S. Speck, Appl. Phys. Lett. 68, 1371 (1996).

${ }^{17}$ R. Armitage, K. Nishizono, J. Suda, and T. Kimoto, J. Cryst. Growth 284, 369 (2005).

${ }^{18}$ I. V. Markov, Crystal Growth for Beginners, Fundamentals of Nucleation, Crystal Growth and Epitaxy, 2nd ed. (World Scientific, Singapore, 2003), p. 172.

${ }^{19}$ I. V. Markov (private communication).

${ }^{20}$ Due to the operational limitation of the plasma unit for $\mathrm{N}$ source, a wider range of growth rate variation was unfeasible.

${ }^{21}$ J. Suda, T. Kurobe, and H. Matsunami, J. Cryst. Growth 201/202, 437 (1999).

${ }^{22}$ M. H. Xie, S. M. Seutter, W. K. Zhu, L. X. Zheng, Huasheng Wu, and S. Y. Tong, Phys. Rev. Lett. 82, 2749 (1999).

${ }^{23}$ M. H. Xie, M. Gong, E. K. Y. Pang, H. S. Wu, and S. Y. Tong, Phys. Rev. B 74, 085314 (2006).

${ }^{24}$ Z. Zhang and H. Metiu, Surf. Sci. 245, 353 (1991). 